\title{
Another Collimation Mechanism of Astrophysical Jet
}

\author{
Yoshinari Minami \\ Advanced Science-Technology Research Organization (Formerly NEC Space Development Division), 35-13, Higashikubo-Cho, \\ Nishi-Ku, Yokohama 220-0062, Japan
}

\begin{abstract}
The acceleration mechanism of astrophysical jet and the collimation mechanism narrowing down to a long distance have been examined so far. It is a collimation problem of how to narrow the astrophysical jet narrowly. Further, the model of the astrophysical jet acceleration mechanism is required to solve this collimation problem at the same time as well as its acceleration. At the present time, the magnetic force model (magnetic centrifugal force and magnetic pressure) is regarded as the most dominant theory which solves the two problems of astrophysical jet acceleration and collimation at the same time. In addition to the present astrophysical jet narrow collimation mechanism by magnetic tension (pinch) force, in this article, another collimation mechanism which narrows down an astrophysical jet is newly introduced. That is, since a curvature is generated in the space around the astrophysical jet by magnetic field, a kind of pressure equivalent to the gravitational effect is generated in the direction of the interior of astrophysical jet as well as the pinch force from the outer circumferential surface of the astrophysical jet.
\end{abstract}

Key words: Astrophysical jet, accretion disk, black hole, collimation mechanism, acceleration mechanism, magnetic field, continuum, curvature, space-time, cosmology.

\section{Introduction}

The astrophysical jet is a narrow jetted plasma jet at high speed $(100 \mathrm{~km} / \mathrm{s}$ to near the speed of light) that emits in both directions vertically from accretion disk around the compact central object such as a neutron star or black hole. Its length is an enormous, long and narrow jet reaching from 1 light year-10 light years - 1 million light years. A jet propagating at a speed close to the speed of light is called a relativistic jet.

The acceleration mechanism of the astrophysical jet and the collimation mechanism narrowing down to a long distance have been examined so far. They are due to thermal gas pressure, light radiation pressure, and magnetic field pressure. Currently, Radiative Acceleration model accelerated by the radiation field of the accretion disk and Magnetic Acceleration model accelerated by magnetic field

Corresponding author: Yoshinari Minami, administrative director, research fields: satellite design and engineering, propulsion theory and propulsion physics, laser propulsion, interstellar navigation theory. E-mail: y-minami@mtj.biglobe.ne.jp. penetrating the accretion disk are representative models. The high velocity, highly collimated gas streams-jets-raise two major problems, namely how the jet material is accelerated, and how it is collimated (Fig. 1a).

It is a collimation problem of how to narrow the jet narrowly, and the model of the jet acceleration mechanism is required to solve this collimation problem at the same time as well as acceleration. At the present time, the magnetic force model (magnetic centrifugal force and magnetic pressure) is regarded as the most dominant theory which solves the two problems of jet acceleration and collimation at the same time. That is, the accretion disk generates a helical magnetic field by twisting the magnetic field lines, accelerates by magnetic force, and narrows the jet by magnetic tension (pinch). The self-pinching force of magnetic field twisted by the rotation occurs naturally as a force to collimate the jet thinly (Fig. 1b) [1-8].

However, there are also controversies among researchers as follows: 1) the global magnetic field cannot be MHD (magnetohydrodynamic) jet due to 


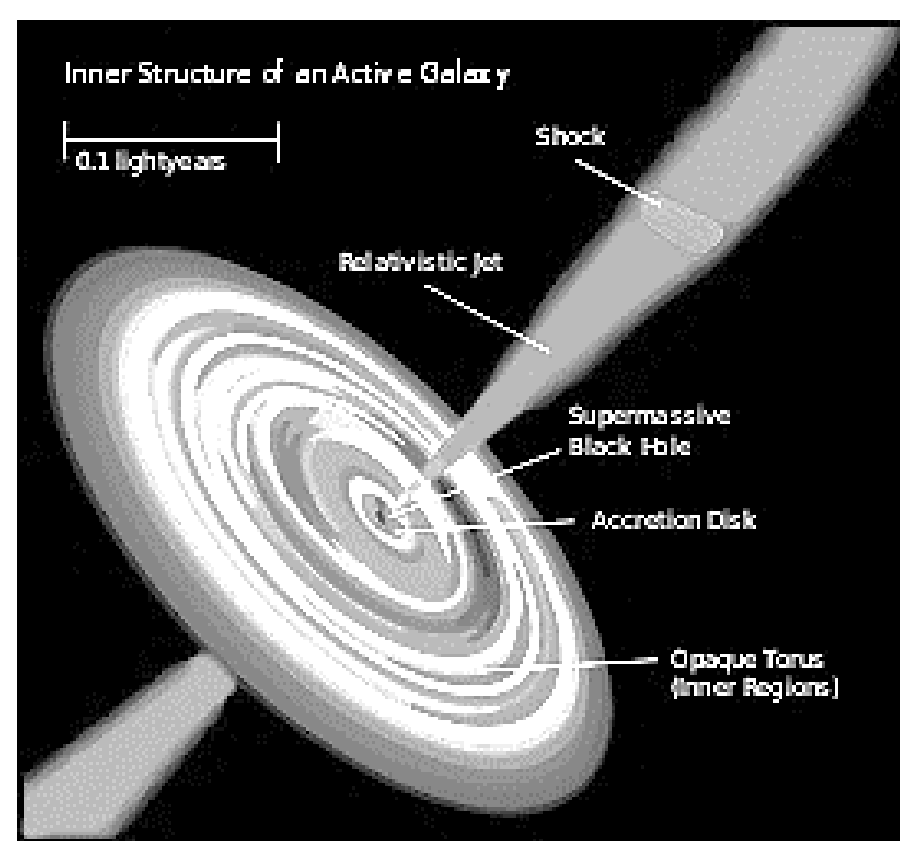

(a)

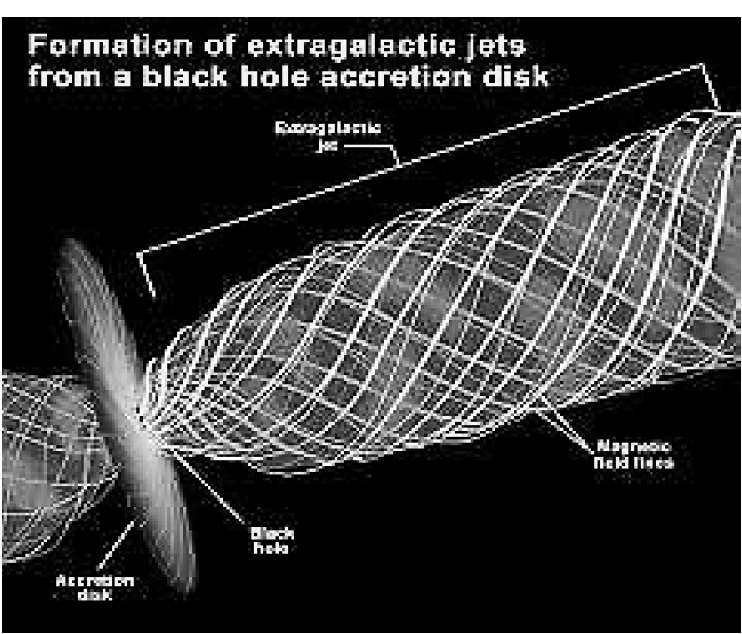

(b)

Fig. 1 (a) Astrophysical Jet from an Accretion Disk of Black Hole; (b) Formation of Astrophysical Jet wound by twisting magnetic field lines.

(http://image.search.yahoo.co.jp/sear ch?rkf=2\&ei=UTF-8\&gdr=1\&p=Astrophysical+Jet)

weak magnetic field; 2) even if the global magnetic field is weak, it can do MHD jet, and the local magnetic field can do MHD jet, furthermore the astrophysical jet can collimate by magnetic field; 3 ) the magnetic field hypothesis is difficult because the magnetic field in the jet is weak.

Even so, if the magnetic field lines are in the jet, there is a possibility that a strong magnetic field region is locally generated due to local turbulence and shock waves in the plasma.

In addition to the conventional jet narrow collimation mechanism by magnetic tension (pinch) force, in this article, we introduce another collimation mechanism which narrows down a jet.

That is, since a magnetic field is present in the jet, a curvature is generated in the surrounding space by magnetic field; a spatial pressure equivalent to the gravitational effect in the surrounding space is generated in the direction of the interior of the jet [9-11]. This spatial pressure acts as well as the pinch force from the outer circumferential surface of the astrophysical jet, i.e., collimation mechanism.

\section{Astrophysical Jet Acceleration and Collimation}

An astrophysical jet is a phenomenon often seen in astronomy, where streams of matter are emitted along the axis of rotation of a compact central object (such as a black hole or neutron star). Many stellar objects with accretion disks have jets. While it is still the subject of ongoing research to understand how jets are formed and powered, the two most often proposed origins are dynamic interactions within the accretion disk, or a process associated with the compact central object. When matter is emitted at speeds approaching the speed of light, these jets are called relativistic jets.

While it is not known exactly how accretion disks would accelerate jets or produce positron-electron plasma, they are generally thought to generate tangled magnetic fields that cause the jets to accelerate and collimate.

One of the astonishing properties of astrophysical jets is that they remain collimated over quite large distances. Again, MHD processes seem to be most 
likely responsible for this behavior: the same pinch mechanism, which forced the plasma gas into a beam directed along the polar axis of the driving source, is also collimating the astrophysical jet further out. The idea of magnetic collimation of jets in the asymptotic regime (i.e., far from the driving sources) has been proposed first for galactic radio jets showed that any axisymmetric (nonrelativistic) magnetized wind will approach a cylindrically collimated structure, if the electric current carried by the flow is non-zero. The collimation mechanism is straightforward to understand for a current carrying flow: the current creates a magnetic field wrapping around the current via Ampère's law. The action of this (toroidal) field then pinches the current back to the flow axis via the Lorentz force. In the case of a vanishing current, the flow would still be paraboloidally collimated. The importance of magnetic fields for jet collimation is valid.

Since the one-fluid approximation holds, the equation of motion of the magnetic fluid is given by

$$
\begin{gathered}
F=J \times B=(\nabla \times B) \times B / \mu_{0} \\
=(B \cdot \nabla) B / \mu_{0}-\nabla\left(B^{2} / 2 \mu_{0}\right)
\end{gathered}
$$

where $F$ is the Lorentz force, $J$ is the current density, $B$ is the magnetic field, $\mu_{0}=4 \pi \times 10^{-7}(\mathrm{H} / \mathrm{m})$.

We use the following equation (Ampère's rule) $\nabla \times B=\mu_{0} J$.
The first term in Eq. (1) denotes magnetic tension and second term denotes magnetic pressure.

Fig. 2a shows cylindrical plasma. A current I flows in the plasma and a magnetic field $B_{\theta}$ occurs in the $\theta$ direction. Although the force of contracting the plasma by $B_{\theta}$ is Lorentz force, magnetic pressure $P_{B}$ is externally applied and the cylindrical plasma is contracted (Fig. 2b).

Using Eqs. (2) and (3),

$$
\begin{gathered}
\int_{S} r o t B d S=\oint_{C} B d s=\int_{S} \mu_{0} J d S=\mu_{0} I \\
\mu_{0} I=\oint_{C} B d s=2 \pi r B_{\theta}
\end{gathered}
$$

magnetic field $\mathrm{B}_{\theta}$ is obtained:

$$
B_{\theta}=\frac{\mu_{0} I}{2 \pi r}
$$

Then, magnetic pressure is obtained:

$$
P_{B}=\frac{B_{\theta}{ }^{2}}{2 \mu_{0}}
$$

Fig. 3a shows General Relativistic MHD simulation on the interaction between ergosphere and magnetic field line of rotating black hole. A black hole dynamics has its plasma aligned to the interstellar magnetic field lines that thread through the equatorial plane of the accretion disk just outside the event horizon, so that the plasma evolves into accretion disk,
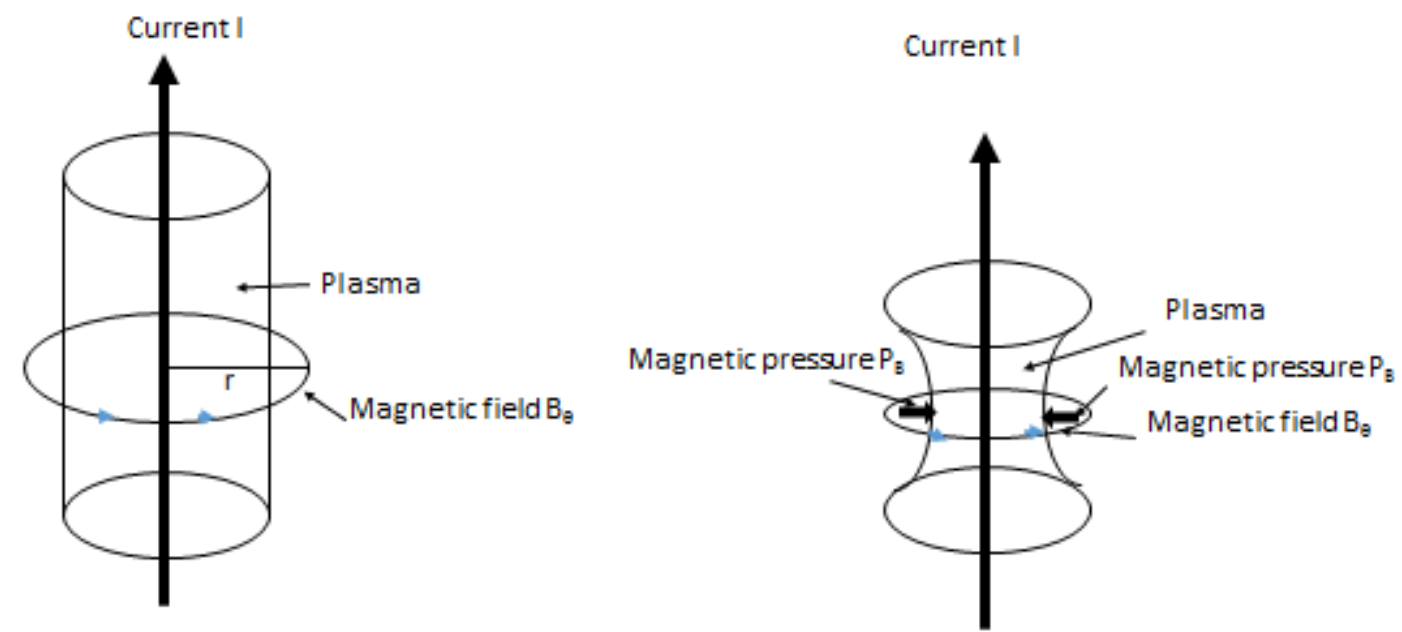

Fig. 2 (a) cylindrical plasma; (b) Contracted cylindrical plasma by magnetic pressure. 


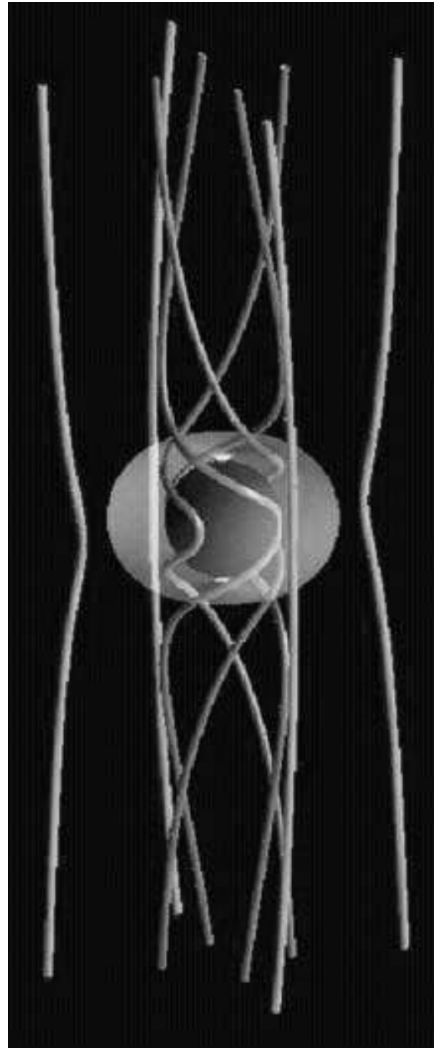

(a)

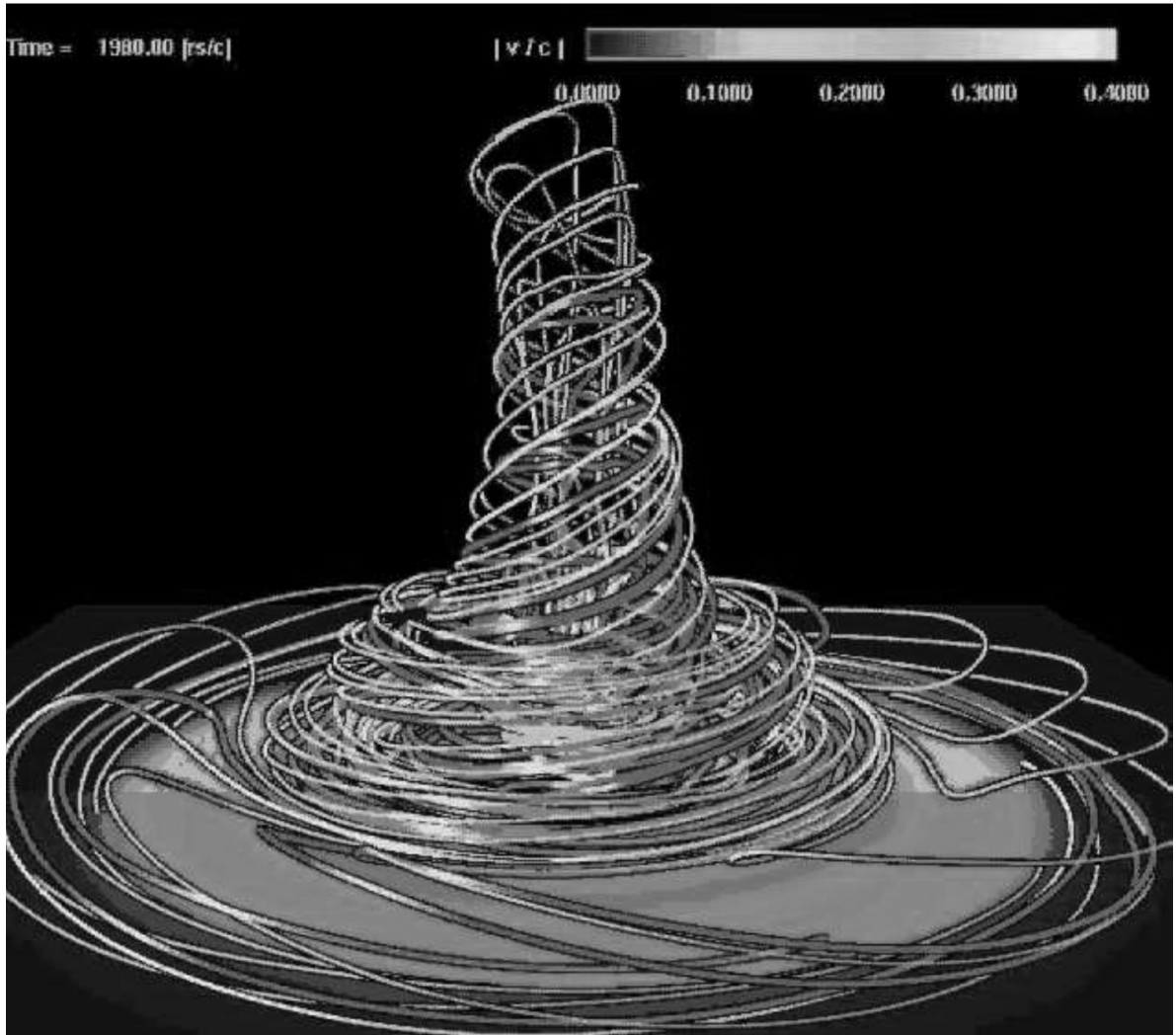

(b)

Fig. 3 (a) MHD jets from Kerr hole magnetosphere (Koide et al. 2002 Science); (b) KatoY, Mineshige, Shibata (2004); 3D sim. (ApJ). This toroidal field dominated jet is launched by magnetic pressure (similar to Shibata and Uchida 1985, Turner et al. 1999, Kudoh et al. 2002), and is also Similar to "magnetic tower" of Lynden-Bell (1996).

which could be described as a condensate of electron-positron pairs. Since the plasma being highly conductive, it will be expected that the interstellar magnetic field lines will become frozen into that plasma which rotates within accretion disk, and as the accretion disk rotates it will drag and twist those magnetic field lines, pulling them together.

Fig. $3 b$ shows a toroidal field dominated jet is launched by magnetic pressure.

As the magnetic field penetrating the accretion disk is twisted, the energy of the magnetic field is accumulated, and at the same time it propagates along the magnetic field lines, the jet ejects from the accretion disk, and not only the magnetic centrifugal force but also magnetic pressure also contributes to acceleration of jet.

When the magnetic field is twisted in the direction of rotation by the actuation rotation of the plasma material, the twisted magnetic field acts like a spring to accelerate the plasma material further upward.

In other words, it is acceleration by magnetic pressure. If the jet is magnetically accelerated, the jet is expected to have a twisted helical magnetic field (Fig. 1b).

Furthermore, the twisted magnetic field acts like a rubber string, and the force of the rubber band shrinks (magnetic pinch) so that the flow of the plasma substance is directed in the rotation axis direction. This is the collimation of the jet by magnetic field. Collimation also occurs voluntarily in addition to acceleration in a model where a jet is driven from an accretion disk by magnetic field and rotation.

Even if the magnetic field penetrating the accretion disk is very weak, the rotation of the accretion disk causes the magnetic field to twist and increase more and more, and the energy is stored in the magnetic 
field to the same extent as the rotational energy of the accretion disk. Even in the case of a local magnetic field in the disk instead of the global magnetic field, the magnetic field is twisted in the disk, so that magnetic pressure is generated and it is possible to accelerate the jet [4-7].

The energy of the magnetic field is increased by compressing the gas or stretching the magnetic field lines due to the plasma gas. The phenomenon in which energy stored in the form of a magnetic field is released locally and in large quantities in a short time is well known for solar flares. The acceleration mechanism for these jets may be similar to the magnetic reconnection processes observed in the Earth's magnetosphere and the solar wind. The energy of magnetic field is given by:

$$
E_{\text {mag }}=\frac{B^{2}}{2 \mu_{0}} \cdot \frac{4 \pi}{3} R^{3}
$$

where $R$ is the radius of sunspot.

As magnetic rotation instability grows, magnetic field lines are stretched in the azimuth direction, the magnetic field is strengthened, and the magnetic energy exponentially increases.

As long as there is a weak magnetic field at the beginning, the magnetic field is amplified by magnetic rotation instability.

\section{Collimation Mechanism of Astrophysical Jet Induced by Spatial Curvature Generated in Space around Magnetic Field}

\subsection{Introduction}

The principle of this idea is derived from General Relativity and the theory of continuum mechanics. We assume that the so-called "vacuum" of space acts as an infinite elastic body like rubber. The curvature of space plays a significant role. A hypothesis for mechanical property of space-time is introduced by Minami in 1988 [11]. Concerning the basic concept, please refer to Appendix A: Continuum Mechanics of Space-Time.
Furthermore, the major component of curvature of space can be produced by not only mass density but also magnetic field.

In the subsequent sections, Generation of Surface Force Induced by Spatial Curvature in the first, Curvature Control by Magnetic Field in the second, and finally, Collimation Mechanism Induced by Spatial Curvature around Magnetic Field are introduced.

\subsection{Generation of Surface Force Induced by Spatial Curvature}

On the supposition that space is an infinite continuum, continuum mechanics can be applied to the so-called "vacuum" of space. This means that space can be considered as a kind of transparent field with elastic properties. Fig. 4 shows the curvature of space.

If space curves, then an inward normal stress "-P" is generated (Fig. 4a). This normal stress, i.e. surface force serves as a sort of pressure field.

$$
-P=N \cdot\left(2 R^{00}\right)^{1 / 2}=N \cdot\left(1 / R_{1}+1 / R_{2}\right)
$$

where $N$ is the line stress, $R_{1}, R_{2}$ are the radius of principal curvature of curved surface, and $R^{00}$ is the major component of spatial curvature.

A large number of curved thin layers form the unidirectional surface force, i.e. acceleration field. Accordingly, the spatial curvature $R^{00}$ produces the acceleration field $\alpha$ (Fig. 4b).

The fundamental three-dimensional space structure is determined by quadratic surface structure. Therefore, a Gaussian curvature $K$ in two-dimensional Riemann space is significant. The relationship between $K$ and the major component of spatial curvature $R^{00}$ is given by:

$$
K=\frac{R_{1212}}{\left(g_{11} g_{22}-g_{12}{ }^{2}\right)}=\frac{1}{2} \cdot R^{00}
$$

where $R_{1212}$ is non-zero component of Riemann curvature tensor.

It is now understood that the membrane force on the curved surface and each principal curvature generates the normal stress "-P" with its direction normal to the 
curved surface as a surface force. The normal stress "-P" acts towards the inside of the surface as shown in Fig. 4a.

A thin-layer of curved surface will take into consideration within a spherical space having a radius of $R$ and the principal radii of curvature that are equal to the radius $\left(R_{1}=R_{2}=R\right)$. Since the membrane force $N$ (serving as the line stress) can be assumed to have a constant value, Eq. (7) indicates that the curvature $R^{00}$ generates the inward normal stress " $-\mathrm{P}$ " of the curved surface. The inwardly directed normal stress serves as a pressure field.

When the curved surfaces are included in a great number, some type of unidirectional pressure field is formed. A region of curved space is made of a large number of curved surfaces and they form the field as a unidirectional surface force (i.e. normal stress). Since the field of the surface force is the field of a kind of force, the force accelerates matter in the field, i.e. we can regard the field of the surface force as the acceleration field. A large number of curved thin layers form the unidirectional acceleration field (Fig. 4b). Accordingly, the spatial curvature $R^{00}$ produces the acceleration field $\alpha$. Therefore, the curvature of space plays a significant role to generate pressure field.

Applying membrane theory, the following equilibrium conditions are obtained in quadratic surface,

$$
N^{\alpha \beta} b_{\alpha \beta}+P=0
$$

where $N^{\alpha \beta}$ is a membrane force, i.e. line stress of curved space, $b_{\alpha \beta}$ is second fundamental metric of
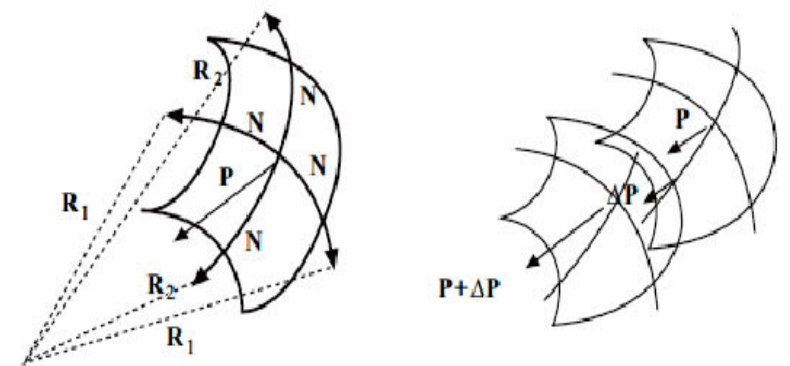

(a) curved surface, and $\mathrm{P}$ is the normal stress on curved surface [9-11, 13-16].

The second fundamental metric of curved space $b_{\alpha \beta}$ and principal curvature $K_{(i)}$ has the following relationship using the metric tensor $g_{\alpha \beta}$,

$$
b_{\alpha \beta}=K_{(i)} g_{\alpha \beta}
$$

Therefore we get:

$$
\begin{gathered}
N^{\alpha \beta} b_{\alpha \beta}=N^{\alpha \beta} K_{(i)} g_{\alpha \beta}= \\
g_{\alpha \beta} N^{\alpha \beta} K_{(i)}=N_{\alpha}{ }^{\alpha} K_{(i)}=N \cdot K_{(i)}
\end{gathered}
$$

From Eqs. (9) and (11), we get:

$$
N_{\alpha}^{\alpha} K_{(i)}=-P
$$

As for the quadratic surface, the indices $\alpha$ and $i$ take two different values, i.e. 1 and 2, therefore Eq. (12) becomes:

$$
N_{1}^{1} K_{(1)}+N_{2}^{2} K_{(2)}=-P
$$

where $K_{(1)}$ and $K_{(2)}$ are principal curvature of curved surface and are inverse number of radius of principal curvature (i.e. $1 / R_{1}$ and $1 / R_{2}$ ).

The Gaussian curvature $\mathrm{K}$ is represented as:

$$
K=K_{(1)} \cdot K_{(2)}=\left(1 / R_{1}\right) \cdot\left(1 / R_{2}\right)
$$

Accordingly, suppose $N_{1}{ }^{1}=N_{2}{ }^{2}=N$, we get:

$$
N \cdot\left(1 / R_{1}+1 / R_{2}\right)=-P
$$

It is now understood that the membrane force on the curved surface and each principal curvature generate the normal stress "-P" with its direction normal to the

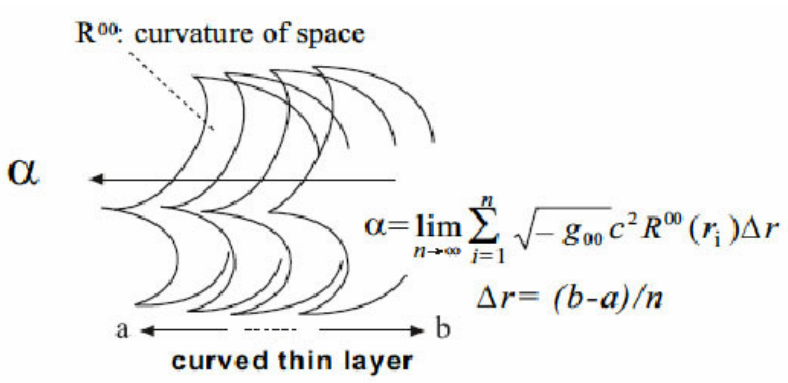

(b)

Fig. 4 Curvature of Space: (a) curvature of space plays a significant role. If space curves, then inward stress (surface force) "P" is generated $\Rightarrow A$ sort of pressure field; (b) a large number of curved thin layers form the unidirectional surface force, i.e. acceleration field $\alpha$. 
curved surface as a surface force. The normal stress "-P" is towards the inside of surface as shown in Fig. 4.

A thin-layer of curved surface will be taken into consideration within a spherical space having a radius of $R$ and the principal radii of curvature which are equal to the radius $\left(R_{1}=R_{2}=R\right)$. From Eqs. (8) and (14), we then get:

$$
K=\frac{1}{R_{1}} \cdot \frac{1}{R_{2}}=\frac{1}{R^{2}}=\frac{R^{00}}{2}
$$

Considering $N \cdot(2 / R)=-P$ of Eq. (15), and substituting Eq. (16) into Eq. (15), the following equation is obtained:

$$
-P=N \cdot \sqrt{2 R^{00}}
$$

Since the membrane force $N$ (serving as the line stress) can be assumed to have a constant value, Eq. (17) indicates that the curvature $R^{00}$ generates the inward normal stress "-P" of the curved surface. The inwardly directed normal stress serves as a kind of pressure field.

Here, we give an account of curvature $R^{00}$ in advance. The solution of metric tensor $g^{\mu \nu}$ is found by gravitational field equation as the following:

$$
R^{\mu v}-\frac{1}{2} \cdot g^{\mu v} R=-\frac{8 \pi G}{c^{4}} \cdot T^{\mu v}
$$

where $R^{\mu v}$ is the Ricci tensor, $R$ is the scalar curvature, $G$ is the gravitational constant, $c$ is the velocity of light, $T^{\mu v}$ is the energy momentum tensor.

Furthermore, we have the following relation for scalar curvature $R$ :

$$
\begin{gathered}
R=R_{\alpha}^{\alpha}=g^{\alpha \beta} R_{\alpha \beta}, R^{\mu \nu}=g^{\mu \alpha} g^{\nu \beta} R_{\alpha \beta}, \\
R_{\alpha \beta}=R^{j}{ }_{\alpha j \beta}=g^{i j} R_{i \alpha j \beta}
\end{gathered}
$$

Ricci tensor $R^{\mu v}$ is represented by:

$$
R_{\mu \nu}=\Gamma_{\mu \alpha, \nu}^{\alpha}-\Gamma_{\mu \nu, \alpha}^{\alpha}-\Gamma_{\mu \nu}^{\alpha} \Gamma_{\alpha \beta}^{\beta}+\Gamma_{\mu \beta}^{\alpha} \Gamma_{v \alpha}^{\beta} \quad\left(=R_{v \mu}\right)
$$

where $\Gamma_{j k}^{i}$ is Riemannian connection coefficient.

If the curvature of space is very small, the term of higher order than the second can be neglected, and Ricci tensor becomes:

$$
R_{\mu v}=\Gamma_{\mu \alpha, v}^{\alpha}-\Gamma_{\mu v, \alpha}^{\alpha}
$$

The major curvature of Ricci tensor $(\mu=v=0)$ is calculated as follows:

$$
R^{00}=g^{00} g^{00} R_{00}=-1 \times-1 \times R_{00}=R_{00}
$$

As previously mentioned, Riemannian geometry is a geometry that deals with a curved Riemann space, therefore Riemann curvature tensor is the principal quantity. All components of Riemann curvature tensor are zero for flat space and non-zero for curved space. If an only non-zero component of Riemann curvature tensor exists, the space is not flat space but curved space. Although Ricci tensor $R^{\mu \nu}$ has 10 independent components, the major component is the case of $\mu=v=0$, i.e., $R^{00}$. Therefore, the major curvature of Ricci tensor $R^{00}$ plays a significant role.

\subsection{Curvature Control by Magnetic Field}

Let us consider the electromagnetic energy tensor $M^{i j}$. In this case, the solution of metric tensor $g_{i j}$ is found by

$$
R^{i j}-\frac{1}{2} \cdot g^{i j} R=-\frac{8 \pi G}{c^{4}} \cdot M^{i j}
$$

Eq. (23) determines the structure of space due to the electromagnetic energy.

Here, if we multiply both sides of Eq. (23) by $g_{i j}$, we obtain

$$
\begin{gathered}
g_{i j}\left(R^{i j}-\frac{1}{2} \cdot g^{i j} R\right)= \\
g_{i j} R^{i j}-\frac{1}{2} \cdot g_{i j} g^{i j} R=R-\frac{1}{2} \cdot 4 R=-R \\
g_{i j}\left(\frac{-8 \pi G}{c^{4}} \cdot M^{i j}\right)= \\
-\frac{8 \pi G}{c^{4}} \cdot g_{i j} M^{i j}=\frac{-8 \pi G}{c^{4}} \cdot M_{i}^{i}=\frac{-8 \pi G}{c^{4}} M
\end{gathered}
$$


The following equation is derived from Eqs. (24) and (25)

$$
R=\frac{8 \pi G}{c^{4}} \cdot M
$$

Substituting Eq. (26) into Eq.(23), we obtain

$$
\begin{array}{r}
R^{i j}=-\frac{8 \pi G}{c^{4}} \cdot M^{i j}+\frac{1}{2} \cdot g^{i j} R= \\
-\frac{8 \pi G}{c^{4}} \cdot\left(M^{i j}-\frac{1}{2} \cdot g^{i j} M\right)
\end{array}
$$

Using antisymmetric tensor $f_{i j}$ which denotes the magnitude of electromagnetic field, the electromagnetic energy tensor $M^{i j}$ is represented as follows;

$$
\begin{gathered}
M^{i j}=-\frac{1}{\mu_{0}} \cdot\left(f^{i \rho} f_{\rho}^{j}-\frac{1}{4} \cdot g^{i j} f^{\alpha \beta} f_{\alpha \beta}\right), \\
f^{i \rho}=g^{i \alpha} g^{\rho \beta} f_{\alpha \beta}
\end{gathered}
$$

Therefore, for $M$, we have

$$
\begin{gathered}
M=M_{i}^{i}=g_{i j} M^{i j}= \\
-\frac{1}{\mu_{0}} \cdot\left(g_{i j} f^{i \rho} f_{\rho}^{j}-\frac{1}{4} \cdot g_{i j} g^{i j} f^{\alpha \beta} f_{\alpha \beta}\right) \\
=-\frac{1}{\mu_{0}} \cdot\left(f^{i \rho} f_{i \rho}-\frac{1}{4} \cdot 4 f^{\alpha \beta} f_{\alpha \beta}\right)= \\
-\frac{1}{\mu_{0}} \cdot\left(f^{i \rho} f_{i \rho}-f^{i \rho} f_{i \rho}\right)=0
\end{gathered}
$$

Accordingly, substituting $M=0$ into Eq. (27), we get

$$
R^{i j}=-\frac{8 \pi G}{c^{4}} \cdot M^{i j}
$$

Although Ricci tensor $R^{i j}$ has 10 independent components, the major component is the case of $i=j=0$, i.e., $R^{00}$. Therefore, Eq. (30) becomes

$$
R^{00}=-\frac{8 \pi G}{c^{4}} \cdot M^{00}
$$

On the other hand, 6 components of antisymmetric tensor $f_{i j}=-f_{j i}$ are given by electric field $\mathrm{E}$ and magnetic field B from the relation to Maxwell's field equations

$$
\begin{gathered}
f_{10}=-f_{01}=\frac{1}{c} \cdot E_{x}, f_{20}=-f_{02}=\frac{1}{c} \cdot E_{y}, \\
f_{30}=-f_{03}=\frac{1}{c} E_{z} \\
f_{12}=-f_{21}=B_{z}, f_{23}=-f_{32}=B_{x}, \\
f_{31}=-f_{13}=B_{y} \\
f_{00}=f_{11}=f_{22}=f_{33}=0
\end{gathered}
$$

Substituting Eq. (32) into Eq. (28), we have

$$
M^{00}=-\left(\frac{1}{2} \cdot \varepsilon_{0} E^{2}+\frac{1}{2 \mu_{0}} \cdot B^{2}\right) \approx-\frac{1}{2 \mu_{0}} \cdot B^{2} .
$$

Finally, from Eqs. (31) and (33), we have

$$
R^{00}=\frac{4 \pi G}{\mu_{0} c^{4}} \cdot B^{2}=8.2 \times 10^{-38} \cdot B^{2} \quad(\text { B in Tesla }),
$$

where we let $\mu_{0}=4 \pi \times 10^{-7}(H / m)$, $\varepsilon_{0}=1 /(36 \pi) \times 10^{-9}(\mathrm{~F} / \mathrm{m}), \quad c=3 \times 10^{8}(\mathrm{~m} / \mathrm{s})$, $G=6.672 \times 10^{-11}\left(N \cdot \mathrm{m}^{2} / \mathrm{kg}^{2}\right), \quad B$ is a magnetic field in Tesla and $R^{00}$ is a major component of spatial curvature $\left(1 / \mathrm{m}^{2}\right)$.

The relationship between curvature and magnetic field was derived by Minami and introduced it in 16th International Symposium on Space Technology and Science (1988) [11]. Eq. (34) is derived from general method using gravitational field equation.

On the other hand, Levi-Civita also investigated the gravitational field produced by a homogeneous electric or magnetic field, which was expressed by Pauli [12]. If $x^{3}$ is taken in the direction of a 
magnetic field of intensity F (Gauss unit), the square of the line element is of the form;

$$
\begin{gathered}
d s^{2}=\left(d x^{1}\right)^{2}+\left(d x^{2}\right)^{2}+\left(d x^{3}\right)^{2} \\
+\frac{\left(x^{1} d x^{1}+x^{2} d x^{2}\right)^{2}}{a^{2}-r^{2}} \\
-\left[c_{1} \exp \left(x^{3} / a\right)+c_{2} \exp \left(-x^{3} / a\right)\right]^{2}\left(d x^{4}\right)^{2}
\end{gathered}
$$

where $\mathrm{r}=\sqrt{\left(x^{1}\right)^{2}+\left(x^{2}\right)^{2}}, c_{1}$ and $c_{2}$ are constants, $a=\frac{c^{2}}{\sqrt{k} F}, \quad k \quad$ is Newtonian gravitational constant $(\mathrm{G})$, and $x^{1} \ldots x^{4}$ are Cartesian coordinates $\left(x^{1} \ldots x^{3}=\right.$ space, $\left.x^{4}=c t\right)$ with orthographic projection.

The space is cylindrically symmetric about the direction of the field, and on each plane perpendicular to the field direction the same geometry holds as in Euclidean space on a sphere of radius $a$, that is, the radius of curvature $a$ is given by

$$
a=\frac{c^{2}}{\sqrt{k} F}
$$

Since the relation of between magnetic field B in SI units and magnetic field $\mathrm{F}$ in CGS Gauss units are described as follows: $B \sqrt{\frac{4 \pi}{\mu_{0}}} \Leftrightarrow F$, then the radius of curvature " $a$ " in Eq. (36) is expressed in SI units as the following (changing symbol, $k \rightarrow G, F \rightarrow B$ ):

$$
\begin{gathered}
a=\frac{c^{2}}{\sqrt{G} F}=\frac{c^{2}}{\sqrt{G} \cdot B \sqrt{\frac{4 \pi}{\mu_{0}}}} \\
\approx\left(3.484 \times 10^{18} \frac{1}{B} \text { meters }\right)
\end{gathered}
$$

While, scalar curvature is represented by

$$
R^{00} \approx R=\frac{1}{a^{2}}=\frac{G B^{2} \frac{4 \pi}{\mu_{0}}}{c^{4}}=\frac{4 \pi G}{\mu_{0} c^{4}} B^{2}
$$

which coincides with Eq. (34).

\subsection{Collimation Mechanism Induced by Spatial Curvature around Magnetic Field}

As mentioned above, the collimation mechanism is summarized as follows.

(1) On the supposition that space is an infinite continuum, continuum mechanics can be applied to the so-called "vacuum" of space. This means that space can be considered as a kind of transparent elastic field. That is, space as a vacuum performs the motion of deformation such as expansion, contraction, elongation, torsion and bending. We can regard space as an infinite elastic body like rubber.

(2) From General Relativity, the major component of curvature of space $R^{00}$ can be produced by not only mass density but also the magnetic field $\mathrm{B}$ as follows (See 3.3 Curvature Control by Magnetic Field):

$$
R^{00}=\frac{4 \pi G}{\mu_{0} c^{4}} \cdot B^{2}=8.2 \times 10^{-38} \cdot B^{2}
$$

Eq. (39) indicates that the major component of spatial curvature can be controlled by magnetic field B.

(3) If space curves, then an inward normal stress "-P" is generated (See 3.2 Generation of Surface Force Induced by Spatial Curvature).

This normal stress, i.e. surface force serves as a sort of a pressure field.

$$
-P=N \cdot\left(2 R^{00}\right)^{1 / 2}=N \cdot\left(1 / R_{1}+1 / R_{2}\right)
$$

where $N$ is the line stress of membrane of curved surface, $R_{1}, R_{2}$ are the radii of principal curvature of curved surface.

A large number of curved thin layers form the unidirectional surface force, i.e. acceleration field. Accordingly, the spatial curvature $R^{00}$ produces the acceleration field $\alpha$.

(4) From the following linear approximation scheme for the gravitational field equation (See 
Appendix B: Acceleration Induced by Spatial Curvature):

(i) weak gravitational field, i.e. small curvature limit, (ii) quasi-static, (iii) slow-motion approximation (i.e. $\mathrm{v} / \mathrm{c}<<1$ ),

we get the following relation between acceleration of curved space and curvature of space:

$$
\alpha^{i}=\sqrt{-g_{00}} c^{2} \int_{a}^{b} R^{00}\left(x^{i}\right) d x^{i}
$$

Eq. (41) indicates that the acceleration field $\alpha^{i}$ is produced in curved space.

(5) In the curved space region, the massive body " $\mathrm{m}$ $(\mathrm{kg})$ " existing in the acceleration field is subjected to the following force $\mathrm{F}^{\mathrm{i}}(\mathrm{N})$ :

Setting $i=3$ (i.e. direction of radius of curvature: $r$ ), we get:

$$
F^{3}=F=m \alpha=m \sqrt{-g_{00}} c^{2} \int_{a}^{b} R^{00}(r) d r
$$

From Eqs. (39), (40) and (42), we obtain the following equations:

$$
\begin{gathered}
-P=N \cdot \sqrt{2 R^{00}}= \\
N \cdot \sqrt{\frac{8 \pi G}{\mu_{0} c^{4}}} \cdot B=4.1 \times 10^{-19} N \cdot B \\
\alpha=\sqrt{-g_{00}} c^{2} \int_{a}^{b} R^{00}(r) d r \approx c^{2} R^{00}(b-a)
\end{gathered}
$$

Eq. (43) indicates the spatial pressure induced by magnetic field. Eq. (44) indicates the acceleration acting on the surface of astrophysical jet. It may be easy to understand by using the spatial pressure as compared with the acceleration.

Next, we describe the pressure of the cylindrical space from the shape of the astrophysical jet.

Fig. 5 shows the spatial pressure or acceleration induced by magnetic field acting on the surface of astrophysical jet.

Fig. 6 shows the spatial pressure as an inward normal stress "-P" about spherical curved space and cylindrical curved space.

As shown in Fig. 6b, the principal radii of curvature $R_{1}=\infty$ and $R_{2}=a$ yield the following:

$$
\begin{gathered}
-P=N \cdot\left(2 R^{00}\right)^{1 / 2}= \\
N \cdot\left(1 / R_{1}+1 / R_{2}\right)=N \cdot 1 / R_{2}=N \cdot 1 / a
\end{gathered}
$$

From Eq. (37),

$$
a=\frac{c^{2}}{\sqrt{G} \cdot B \sqrt{\frac{4 \pi}{\mu_{0}}}}=\sqrt{\frac{\mu_{0} c^{4}}{4 \pi G}} \cdot \frac{1}{B}
$$

Then we get

$$
-P=N \cdot \frac{1}{a}=\sqrt{\frac{4 \pi G}{\mu_{0} c^{4}}} N \cdot B
$$

Accordingly, spatial pressure (normal stress) from the concentric spatial surface surrounding the jet collimates the jet.

However, since the shape and intensity of the magnetic field change depending on the turbulence of the plasma and the condition of the shock wave inside the astrophysical jet, it may be possible to apply a

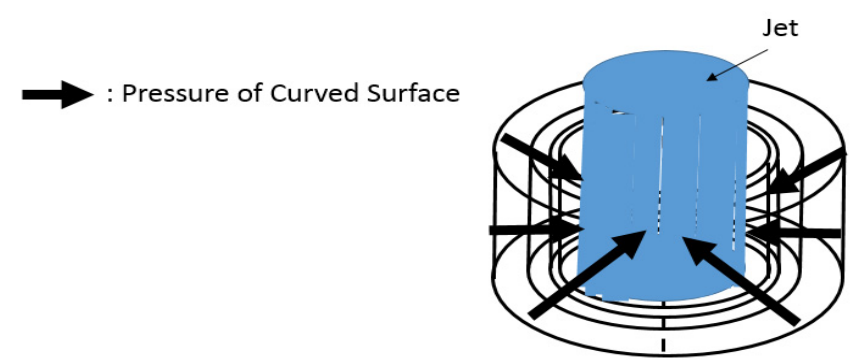

Spatial pressure (normal stress) from the concentric spatial surface

(a)

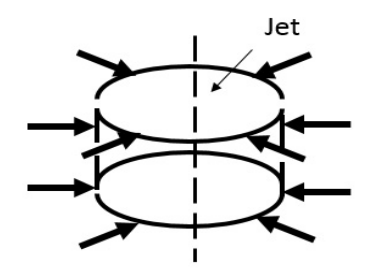

(b)

Fig. 5 (a) Spatial pressure from the concentrically curved multilayer space to the vicinity surface of the cylinder jet; (b) Extended figure of (a). 

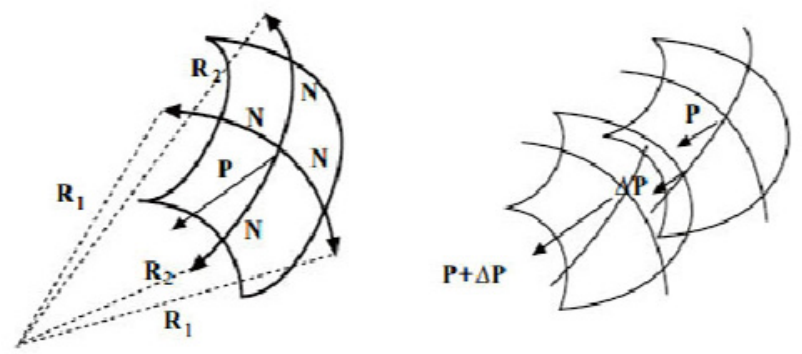

(a)
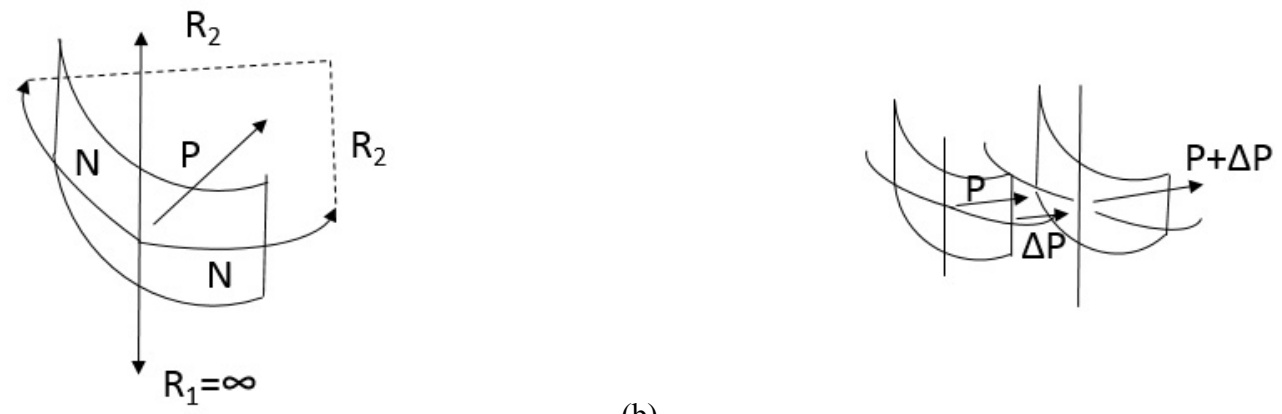

(b)

Fig. 6 (a) Spherical curved space. If space curves, then inward stress (surface force) "P" is generated $\Rightarrow A$ sort of pressure field; (b) Cylindrical curved space for Astrophysical Jet.

spherical curved space (Fig. 6a) rather than cylindrical curved space (Fig. 6b).

Since the present space is rigid, the line stress of space "N" seems to be expected as large value.

\section{Conclusion}

Magnetic force model (magnetic centrifugal force and magnetic pressure) is regarded as the most dominant theory which solves the two problems of astrophysical jet acceleration and collimation at the same time. That is, the accretion disk generates a helical magnetic field by twisting the magnetic field lines, accelerates by magnetic force, and narrows the jet by magnetic tension (pinch).

On the other hand, since the magnetic field is present in the astrophysical jet, a spatial curvature is generated induced by magnetic field in the surrounding space; a spatial pressure in space equivalent to the gravitational effect is generated in the direction of the interior of the astrophysical jet as well as the pinch force from the outer circumferential surface of the astrophysical jet.
Accordingly, although its effect may be small than magnetic pressure, another collimation mechanism of astrophysical jet can be possible to exist.

\section{References}

[1] Contopoulos, I., Gabuzda, D., and Kylafis, N., eds. 2015. The Formation and Disruption of Black Hole Jets. Springer.

[2] Dermer, C. D., and Menon, G. 2009. High Energy Radiation from Black Holes. Princeton University Press.

[3] Kato, S., Fukue, J., and Mineshige, S. 2008. Black-Hole Accretion Disks-Towards a New Paradigm. Kyoto University Press.

[4] Shibata, K., Fukue, J., Matsumoto, R., and Mineshige, S., eds. 1999. Active Universe-Physics of Activity in Astrophysical Objects. Tokyo: Shokabo.

[5] Fukue, J. 2007. Shining Black-Hole Accretion Disks. Pleiades Publishing Co. Ltd.

[6] Mineshige, S. 2016. Black Hole Astrophysics. Nippon Hyoron sha Co. Ltd.

[7] Koyama, K., and Mineshige, S. 2007. Black Hole and High-Energy Phenomena, Nippon Hyoron sha Co., Ltd.

[8] Minami, Y. 2016. "A Journey to the Stars: Space Propulsion Brought about by Astrophysical Phenomena Such as Accretion Disk and Astrophysical Jet." Global Journal of Technology \& Optimization 7: 2. doi: 
10.4172/2229-8711.1000197.

[9] Minami, Y. 2015. "Continuum Mechanics of Space Seen from the Aspect of General Relativity-An Interpretation of the Gravity Mechanism." Journal of Earth Science and Engineering 5: 188-202.

[10] Williams, C., (ed.); Minami, Y., (Chap. 3), et al. 2015. Advances in General Relativity Research. Nova Science Publishers.

[11] Minami, Y. 1988. "Space Strain Propulsion System." 16th International Symposium on Space Technology and Science (16th ISTS), Vol. 1, 125-36.
[12] Pauli, W. 1981. Theory of Relativity. New York: Dover Publications, Inc.

[13] Flügge, W. 1972. Tensor Analysis and Continuum Mechanics. Berlin, Heidelberg, New York: Springer-Verlag.

[14] Fung, Y. C. 2001. Classical and Computational Solid Mechanics. World Scientific Publishing Co. Pre. Ltd.

[15] Hans, Z. 1977. An Introduction to Thermomechanics. North-Holland Publishing Company.

[16] Borg, S. F. 1963. Matrix-Tensor Methods in Continuum Mechanics. D. Van Nostrand Company. 


\section{Appendix A: Continuum Mechanics of Space-Time}

Given a priori assumption that space as a vacuum has a physical fine structure like continuum, it enables us to apply a continuum mechanics to the so-called "vacuum" of space. Assuming that space as vacuum is an infinite continuum, space can be considered as a kind of transparent elastic field, and its structure is determined by Riemannian geometry. That is, space as a vacuum performs the motions of deformation such as expansion, contraction, elongation, torsion and bending. The latest expanding universe theories (Friedmann, de Sitter, inflationary cosmological model) support this assumption.

Since the subject of our study is a four-dimensional Riemann space as a curved space, we ascribe a great deal of importance to the curvature of space. We a priori accept that the nature of actual physical space is a four-dimensional Riemann space, that is, three dimensional space $\left(x=x^{1}, y=x^{2}, z=x^{3}\right)$ and one dimensional time $\left(w=c t=x^{0}\right)$, where $c$ is the velocity of light. These four coordinate axes are denoted as $\mathrm{x}^{\mathrm{i}}(i=0,1,2,3)$.

The square of the infinitesimal distance "ds" between two infinitely proximate points $x^{i}$ and $x^{i}+d x^{i}$ is given by equation of the form:

$$
d s^{2}=g_{i j} d x^{i} d x^{j}
$$

where $\mathrm{g}_{\mathrm{ij}}$ is a metric tensor.

The metric tensor $g_{\mathrm{ij}}$ determines all the geometrical properties of space and it is a function of this space coordinate. In Riemann space, the metric tensor $\mathrm{g}_{\mathrm{ij}}$ determines a Riemannian connection coefficient $\Gamma_{j k}^{i}$, and furthermore determines the Riemann curvature tensor $R_{i j k}^{p} \quad$ or $R_{p i j k}$, thus the geometry of space is determined by a metric tensor.

Riemannian geometry is a geometry which provides a tool to describe curved Riemann space, therefore a Riemann curvature tensor is the principal quantity. All the components of Riemann curvature tensor are zero for flat space and non-zero for curved space. If a non-zero component of Riemann curvature tensor exists, the space is not flat space, but curved space. In curved space, it is well known that the result of the parallel displacement of vector depends on the choice of the path. Further, the components of a vector differ from the initial value, after we displace a vector parallel along a closed curve until it returns to the starting point.

An external physical action such as the existence of mass energy or electromagnetic energy yields the structural deformation of space. In the deformed space region, the infinitesimal distance is given by:

$$
d s^{\prime 2}=g_{i j}^{\prime} d x^{i} d x^{j}
$$

where $g_{i j}^{\prime}$ the metric tensor of deformed space region, and we use the convected coordinates $\left(x^{\prime i}=x^{i}\right)$.

As shown in Fig. A, if the line element between the arbitrary two near points (A and B) in space region $\boldsymbol{S}$ (before structural deformation) is defined as $d s=g_{i} d x^{i}$, the infinitesimal distance between the two near points is given by Eq. (A1): $d s^{2}=g_{i j} d x^{i} d x^{j}$.

Let us assume that a space region $\mathbf{S}$ is structurally deformed by an external physical action and transformed to space region $\mathbf{T}$. In the deformed space region $\mathbf{T}$, the line element between the identical two near point (A' and B') of the identical space region newly changes, differs from the length and direction, and becomes $d s^{\prime}=g_{i}^{\prime} d x^{i}$. 


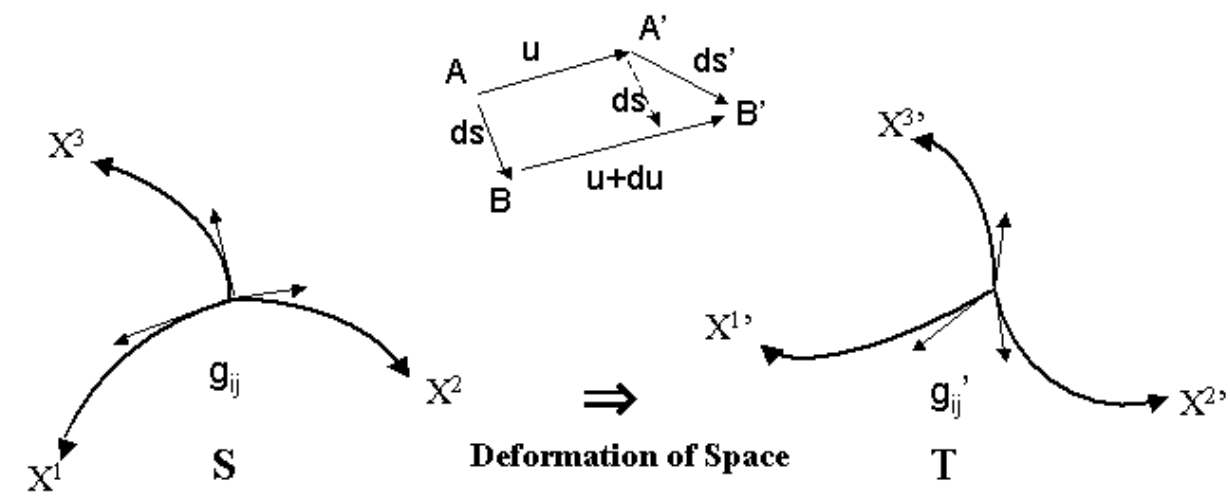

Fig. A Fundamental structure of Space.

Therefore, the infinitesimal distance between the two near points using the convected coordinate $\left(x^{\prime i}=x^{i}\right)$ is given by:

$$
d s^{\prime 2}=g_{i j}^{\prime} d x^{i} d x^{j}
$$

The $g_{i}^{\prime}$ is the transformed base vector from the original base vector $g^{i}$ and the $g_{i j}^{\prime}$ is the transformed metric tensor from the original metric tensor $g_{i j}$. Since the degree of deformation can be expressed as the change of distance between the two points, we get:

$$
d s^{\prime 2}-d s^{2}=g_{i j}^{\prime} d x^{i} d x^{j}-g_{i j} d x^{i} d x^{j}=\left(g_{i j}^{\prime}-g_{i j}\right) d x^{i} d x^{j}=r_{i j} d x^{i} d x^{j}
$$

Hence the degree of geometrical and structural deformation can be expressed by the quantity denoted change of metric tensor, i.e.

$$
r_{i j}=g_{i j}^{\prime}-g_{i j}
$$

On the other hand, the state of deformation can be also expressed by the displacement vector " $u$ " (see Fig. A).

From the continuum mechanics [13-16], using the following equations:

$$
\begin{gathered}
d u=g^{i} u_{i: j} d x^{j} \\
d s^{\prime}=d s+d u=d s+g^{i} u_{i: j} d x^{j}
\end{gathered}
$$

Here we use the usual notation ":?" for covariant differentiation. As is well known, the partial derivative $u_{i, j}=\frac{\partial u_{i}}{\partial x^{j}}$ is not tensor equation. The covariant derivative $u_{i: j}=u_{i, j}-u_{k} \Gamma_{i j}^{k}$ is tensor equation and can be carried over into all coordinate systems.

From usual continuum mechanics, the infinitesimal distance after deformation becomes as follow [13]:

$$
d s^{\prime 2}-d s^{2}=r_{i j} d x^{i} d x^{j}=\left(u_{i: j}+u_{j: i}+u^{k}: u_{k: j}\right) d x^{i} d x^{j}
$$

The terms of higher order than second $u^{k}: i u_{k: j}$ can be neglected if the displacement is of small enough value. As the actual physical space can be dealt with the minute displacement from the trial calculation of strain, we get:

$$
r_{i j}=u_{i: j}+u_{j: i}
$$

Whereas, according to the continuum mechanics [13], the strain tensor $e_{i j}$ is given by: 


$$
e_{i j}=\frac{1}{2} \cdot r_{i j}=\frac{1}{2} \cdot\left(u_{i: j}+u_{j: i}\right)
$$

So, using Eqs. (A5) and (A10) we get:

$$
d s^{2}-d s^{2}=\left(g_{i j}^{\prime}-g_{i j}\right) d x^{i} d x^{j}=2 e_{i j} d x^{i} d x^{j}
$$

where $g_{i j}^{\prime}, g_{i j}$ is a metric tensor, $e_{i j}$ is a strain tensor, and $d s^{\prime 2}-d s^{2}$ is the square of the infinitesimal distance between two infinitely proximate points $x^{i}$ and $x^{i}+d x^{i}$.

From Eq. (A11), the strain of space is described as follows:

$$
e_{i j}=1 / 2 \cdot\left(g_{i j}{ }^{\prime}-g_{i j}\right)
$$

Eq. (A12) indicates that a certain geometrical structural deformation of space is shown by the concept of strain. In essence, the change of metric tensor $\left(g_{i j}^{\prime}-g_{i j}\right)$ due to the existence of mass energy or electromagnetic energy tensor produces the strain field $e_{i j}$. Namely, a certain structural deformation of space-time is described by strain tensor $e_{i j}$; the physical strain is generated by the difference of a geometrical metric of space-time.

\section{Appendix B: Acceleration Induced by Spatial Curvature}

A massive body causes the curvature of space-time around it, and a free particle responds by moving along a geodesic in that space-time. The path of free particle is a geodesic line in space-time and is given by the following geodesic equation:

$$
\frac{d^{2} x^{i}}{d \tau^{2}}+\Gamma_{j k}^{i} \cdot \frac{d x^{j}}{d \tau} \cdot \frac{d x^{k}}{d \tau}=0
$$

where $\Gamma_{j k}^{i}$ is Riemannian connection coefficient, $\tau$ is proper time, $x^{i}$ is four-dimensional Riemann space, that is, three dimensional space $\left(\mathrm{x}=\mathrm{x}^{1}, \mathrm{y}=\mathrm{x}^{2}, \mathrm{z}=\mathrm{x}^{3}\right)$ and one dimensional time $\left(\mathrm{w}=\mathrm{ct}=\mathrm{x}^{0}\right)$, where $\mathrm{c}$ is the velocity of light. These four coordinate axes are denoted as $\mathrm{x}^{\mathrm{i}}(i=0,1,2,3)$.

Proper time is the time to be measured in a clock resting for a coordinate system. We have the following relation described from an invariant line element $d s^{2}$ between Special Relativity (flat space) and General Relativity (curved space):

$$
d \tau=\sqrt{-g_{00}} d x^{0}=\sqrt{-g_{00}} c d t
$$

From Eq. (B1), the acceleration of free particle is obtained by

$$
\alpha^{i}=\frac{d^{2} x^{i}}{d \tau^{2}}=-\Gamma_{j k}^{i} \cdot \frac{d x^{j}}{d \tau} \cdot \frac{d x^{k}}{d \tau}
$$

As is well known in General Relativity, in the curved space region, the massive body " $m(\mathrm{~kg})$ )" existing in the acceleration field is subjected to the following force $F^{i}(\mathrm{~N})$ :

$$
F^{i}=m \Gamma_{j k}^{i} \cdot \frac{d x^{j}}{d \tau} \cdot \frac{d x^{k}}{d \tau}=m \sqrt{-g_{00}} c^{2} \Gamma_{j k}^{i} u^{j} u^{k}=m \alpha^{i}
$$

where $u^{j}, u^{k}$ are the four velocity, $\Gamma_{j k}^{i}$ is the Riemannian connection coefficient, and $\tau$ is the proper time.

From Eqs. (B3) and (B4), we obtain: 


$$
\alpha^{i}=\frac{d^{2} x^{i}}{d \tau^{2}}=-\Gamma_{j k}^{i} \cdot \frac{d x^{j}}{d \tau} \cdot \frac{d x^{k}}{d \tau}=-\sqrt{-g_{00}} c^{2} \Gamma_{j k}^{i} u^{j} u^{k}
$$

Eq. (B5) yields a more simple equation from the condition of linear approximation, that is, weak-field, quasi-static, and slow motion (speed $\mathrm{v}<<$ speed of light $c: u^{0} \approx 1$ ):

$$
\alpha^{i}=-\sqrt{-g_{00}} \cdot c^{2} \Gamma_{00}^{i}
$$

On the other hand, the major component of spatial curvature $R^{00}$ in the weak field is given by

$$
R^{00} \approx R_{00}=R_{0 \mu 0}^{\mu}=\partial_{0} \Gamma_{0 \mu}^{\mu}-\partial_{\mu} \Gamma_{00}^{\mu}+\Gamma_{0 \mu}^{v} \Gamma_{v 0}^{\mu}-\Gamma_{00}^{v} \Gamma_{v \mu}^{\mu}
$$

In the nearly Cartesian coordinate system, the value of $\Gamma_{v \rho}^{\mu}$ are small, so we can neglect the last two terms in Eq. (B7), and using the quasi-static condition we get

$$
R^{00} \approx-\partial_{\mu} \Gamma_{00}^{\mu}=-\partial_{i} \Gamma_{00}^{i}
$$

From Eq. (B8), we get formally

$$
\Gamma_{00}^{i}=-\int R^{00}\left(x^{i}\right) d x^{i}
$$

Substituting Eq. (B9) into Eq. (B6), we obtain

$$
\alpha^{i}=\sqrt{-g_{00}} c^{2} \int_{a}^{b} R^{00}\left(x^{i}\right) d x^{i}
$$

where $\alpha^{i}$ : acceleration $\left(\mathrm{m} / \mathrm{s}^{2}\right), g_{00}$ : time component of metric tensor, a-b: range of curved space region $(\mathrm{m}), x^{i}$ : components of coordinate $(i=0,1,2,3), c$ : velocity of light, $R^{00}$ : major component of spatial curvature $\left(1 / \mathrm{m}^{2}\right)$.

Eq. (B10) indicates that the acceleration field $\alpha^{i}$ is produced in curved space. The intensity of acceleration produced in curved space is proportional to the product of spatial curvature $R^{00}$ and the length of curved region.

Eq. (B4) yields more simple equation from above-stated linear approximation $\left(u^{0} \approx 1\right)$,

$$
F^{i}=m \sqrt{-g_{00}} c^{2} \Gamma_{00}^{i} u^{0} u^{0}=m \sqrt{-g_{00}} c^{2} \Gamma_{00}^{i}=m \alpha^{i}=m \sqrt{-g_{00}} c^{2} \int_{a}^{b} R^{00}\left(x^{i}\right) d x^{i}
$$

Setting $i=3$ (i.e., direction of radius of curvature: $r$ ), we get Newton's second law:

$$
F^{3}=F=m \alpha=m \sqrt{-g_{00}} c^{2} \int_{a}^{b} R^{00}(r) d r=m \sqrt{-g_{00}} c^{2} \Gamma_{00}^{3}
$$

The acceleration $(\alpha)$ of curved space and its Riemannian connection coefficient $\left(\Gamma_{00}^{3}\right)$ are given by:

$$
\alpha=\sqrt{-g_{00}} c^{2} \Gamma_{00}^{3}, \quad \Gamma_{00}^{3}=\frac{-g_{00,3}}{2 g_{33}}
$$

where $c$ : velocity of light, $g_{00}$ and $g_{33}$ : component of metric tensor, $g_{00,3}: \partial g_{00} / \partial x^{3}=\partial g_{00} / \partial r$. We choose the spherical coordinates " $c t=$ $x^{0}, r=x^{3}, \theta=x^{1}, \varphi=x^{2}$ " in space-time. The acceleration $\alpha$ is represented by the equation both in the differential form and in the integral form. Practically, since the metric is usually given by the solution of gravitational field equation, the differential form has been found to be advantageous. 